\title{
AVERAGES IN VECTOR SPACES OVER FINITE FIELDS
}

\author{
ANTHONY CARBERY, BRENDAN STONES AND JAMES WRIGHT
}

\begin{abstract}
We study the analogues of the problems of averages and maximal averages over a surface in $\mathbb{R}^{n}$ when the euclidean structure is replaced by that of a vector space over a finite field, and obtain optimal results in a number of model cases.
\end{abstract}

\section{INTRODUCTION}

In this paper we study the analogues of the problems of averages and maximal averages over a surface in $\mathbb{R}^{n}$ when the euclidean structure is replaced by that of a vector space over a finite field. This point of view has proved beneficial in other problems in harmonic analysis where curvature plays a role and where the underlying question is a local one, such as in the restriction problem for the Fourier transform and in estimates for the Kakeya maximal function, (see for example [12], $[5]$ and [1]).

In order to formulate our results we need some notation. Let $\mathbb{F}$ be a finite field of characteristic $q>2$. Then $\mathbb{F}^{n}$ is a (locally) compact abelian group and as such has a Fourier analysis. In particular, $\mathbb{F}^{n}$ has dual group $\mathbb{F}^{n *}$ (isomorphic to $\mathbb{F}^{n}$ ) which we endow with normalised counting measure $d \xi$ so that for $f: \mathbb{F}^{n *} \rightarrow \mathbb{C}$, and $1 \leq p<\infty$

$$
\begin{aligned}
\|f\|_{p} & =\left(\int_{\xi \in \mathbb{F}^{n *}}|f(\xi)|^{p} d \xi\right)^{\frac{1}{p}} \\
& :=\left(\frac{1}{\mid \mathbb{F}^{n}} \sum_{\xi \in \mathbb{F}^{n *}}|f(\xi)|^{p}\right)^{\frac{1}{p}}
\end{aligned}
$$

All authors were supported by the EC project "HARP". The first author was also partly supported by a Leverhulme Study Abroad Fellowship and EC project "Pythagoras". The third author was also partly supported by an EPSRC grant. 
(and when $p=\infty,\|f\|_{\infty}=\max _{\xi \in \mathbb{F}^{n *}}|f(\xi)|$ ). If $\sigma$ is a measure on $\mathbb{F}^{n *}$ defined via its action on a function $\phi$ by

$$
\begin{aligned}
\langle\phi, \sigma\rangle & =\int_{\xi \in \mathbb{F}^{n *}} \phi(\xi) d \sigma(\xi) \\
& =\frac{1}{|\mathbb{F}|^{n}} \sum_{\xi \in \mathbb{F}^{n *}} \phi(\xi) w(\xi)
\end{aligned}
$$

we identify $\sigma$ with the function $w$. In particular, if $\mathfrak{p}: \mathbb{F}^{k} \rightarrow \mathbb{F}^{n *}$ parametrises a " $k$-dimensional surface in $\mathbb{F}^{n *}$, then the "surface measure" $\sigma_{\mathfrak{p}}$ associated to $\mathfrak{p}$ is given by

$$
\begin{aligned}
\left\langle\phi, \sigma_{\mathfrak{p}}\right\rangle & =\frac{1}{|\mathbb{F}|^{k}} \sum_{s \in \mathbb{F}^{k}} \phi(\mathfrak{p}(s)) \\
& =\frac{1}{|\mathbb{F}|^{k}} \sum_{\xi \in \mathbb{F}^{n *}} \phi(\xi) \# \mathfrak{p}^{-1}(\xi) .
\end{aligned}
$$

Thus the measure $\sigma_{\mathfrak{p}}$ is associated with the function $w(\xi)=|\mathbb{F}|^{n-k} \# \mathfrak{p}^{-1}(\xi)$. (Note that the total mass of $\sigma_{\mathfrak{p}}$ is 1 .)

Convolution of two functions $f$ and $g$ on $\mathbb{F}^{n *}$ is given by

$$
\begin{aligned}
f * g(\xi) & =\int_{\mathbb{F}^{n *}} f(\xi-\eta) g(\eta) d \eta \\
& =\frac{1}{\mid \mathbb{F}^{n}} \sum_{\eta \in \mathbb{F}^{n *}} f(\xi-\eta) g(\eta) .
\end{aligned}
$$

Our first basic object of study will be the averaging operator associated to a $k$ dimensional polynomial surface in $\mathbb{F}^{n *}$. Let $\mathfrak{p}: \mathbb{F}^{k} \rightarrow \mathbb{F}^{n *}$ be a vector-valued polynomial of degree at most $d$. (By the degree of a vector-valued polynomial we mean the maximum of the degrees of its components.) To $\mathfrak{p}$ we associate the normalised surface measure $\sigma_{\mathfrak{p}}$ as above. Then the average of $f: \mathbb{F}^{n *} \rightarrow \mathbb{C}$ along $\mathfrak{p}$ at $\xi$ is $f * \sigma_{\mathfrak{p}}(\xi)$ and we are interested in the mapping properties of

$$
f \mapsto f * \sigma_{\mathfrak{p}}
$$

with respect to the $L^{p}$-norms on $\mathbb{F}^{n *}$ defined above.

For the second object of study we fix $d$, and have an indexing set $\mathcal{A}$ and, for each $\alpha \in \mathcal{A}$, a polynomial $\mathfrak{p}_{\alpha}: \mathbb{F}^{k} \rightarrow \mathbb{F}^{n *}$ of degree at most $d$. We consider the maximal averaging operator

$$
f \mapsto \sup _{\alpha \in \mathcal{A}}\left|f * \sigma_{\mathfrak{p}_{\alpha}}\right|
$$

Once again we wish to examine the mapping properties of this operator with respect to the $L^{p}$-norms.

As all sets and sums are finite, there is no question of the boundedness a priori of these operators between any $L^{p}$-spaces. What interests us here is the possibility of bounds which, for example, depend only upon $k, n, d$ and $\max _{\xi \in \mathbb{F}^{n *}} \# \mathfrak{p}^{-1}(\xi)$ in the first case above, and, in any case, are independent of $|\mathbb{F}|$. 
In this we are obviously motivated by the corresponding euclidean problems. In that setting, $\sigma$ is a finite measure associated to a compact piece of $k$-dimensional surface in $\mathbb{R}^{n}$; convolution with $\sigma$ is then a local operation and can be thought of taking place on chunks of $\mathbb{R}^{n}$ (cubes, balls etc.) of finite volume. So we may as well be working with measures $\sigma$ of unit mass supported in the unit cube in $\mathbb{R}^{n}$ of mass one, and functions similarly supported. This explains the choice of normalisations that we use. Furthermore, considering Riemann sums for the euclidean convolutions and $L^{p}$-norm evaluations as approximations to the genuine article, one of course wants estimates independent of the fineness of the mesh involved. In our current case the "fineness of the mesh" is measured by the quantity $|\mathbb{F}|^{-1}$, and so we are consciously seeking estimates which do not explicitly depend on $|\mathbb{F}|$.

In the euclidean case these problems have a rich history (see [9]) but a complete resolution is seemingly still some way off. Some further partial results are known, especially when the surface is either 1 - or $(n-1)$ - dimensional. See [3], [7], [8], and [10]. In the intermediate range of dimensions $2 \leq k \leq n-2$ matters seem far from being fully understood.

The main point of this paper is to exhibit, for each $1 \leq k \leq n-1$, nontrivial $k$-dimensional surfaces in $\mathbb{F}^{n *}$ for which the averaging and maximal averaging problems admit a complete solution. For $1 \leq k \leq n-1$, define $\mathfrak{p}_{k}: \mathbb{F}^{k} \rightarrow \mathbb{F}^{n}$ by

$\mathfrak{p}_{k}(t)=\left(t_{1}, t_{2}, \ldots ., t_{k}, t_{1}^{2}+t_{2}^{2}+\ldots . .+t_{k}^{2}, t_{1}^{3}+\ldots+t_{k}^{3}, \ldots \ldots \ldots, t_{1}^{n-k+1}+\ldots \ldots+t_{k}^{n-k+1}\right)$.

Note that $\mathfrak{p}_{k}$ "interpolates" between the paraboloid when $k=n-1$ and the curve $\mathfrak{p}_{1}(t)=\left(t, t^{2}, \ldots, t^{n}\right)$ when $k=1$.

Theorem 1. Let $1 \leq k \leq n-1$, and let $\mathbb{F}$ be a finite field of charactersistic greater than $n-k+1$. Let $1 \leq p, q \leq \infty$. Then there is a constant $C$ independent of $|\mathbb{F}|$ such that

$$
\left\|f * \sigma_{\mathfrak{p}_{k}}\right\|_{L^{q}\left(\mathbb{F}^{n *}\right)} \leq C\|f\|_{L^{p}\left(\mathbb{F}^{n *}\right)}
$$

if and only if $(1 / p, 1 / q)$ lies in the convex hull of $(0,0),(1,1),(0,1)$ and $\left(\frac{k}{2 n-k}, \frac{n-k}{2 n-k}\right)$. Furthermore, if $(1 / p, 1 / q)=\left(\frac{k}{2 n-k}, \frac{n-k}{2 n-k}\right)$ we may take the constant $C$ to be $1+$ $(n-k)^{\frac{2 k(n-k)}{2 n-k}}$.

For the next result we need to define a suitable family over which we can take maximal averages. We first note that inequality (1) is invariant under affine transformations, so it is reasonable to build a maximal function over a family of affinely equivalent convolutions. Let $\mathcal{A}$ be an indexing set with $\# \mathcal{A}=|\mathbb{F}|^{r}$ for some $0 \leq r \leq n-k$. For $\alpha \in \mathcal{A}$, let $A_{\alpha}$ be an invertible $n \times n$ matrix over $\mathbb{F}$ and let $b_{\alpha}$ be a vector in $\mathbb{F}^{n}$. Define $\mathfrak{p}_{k, \alpha}$ to be $A_{\alpha} \mathfrak{p}_{k}+b_{\alpha}$. Thus $\mathfrak{p}_{k, \alpha}$ is just an affine transform of $\mathfrak{p}_{k}$. In the statement of Theorem 2 below, we suppose (for simplicity) that the images of the $\mathfrak{p}_{k, \alpha}$ as $\alpha$ ranges over $\mathcal{A}$ are disjoint.

Theorem 2. Let $1 \leq k \leq n-1$, and let $\mathbb{F}$ be a finite field of charactersistic greater than $n-k+1$. Let $p \geq 1$. Let $r$ be an integer such that $0 \leq r \leq n-k$. Then there exists a constant $C$ independent of $|\mathbb{F}|$ such that whenever $\mathcal{A}$ is an indexing set of 
cardinality $|\mathbb{F}|^{r}$, whenever $\left\{A_{\alpha}: \alpha \in \mathcal{A}\right\}$ is a collection of invertible matrices and whenever $\left\{b_{\alpha}: \alpha \in \mathcal{A}\right\}$ is a collection of vectors,

$$
\left\|\sup _{\alpha \in \mathcal{A}}\left|f * \sigma_{\mathfrak{p}_{k, \alpha}}\right|\right\|_{L^{p}\left(\mathbb{F}^{* n}\right)} \leq C\|f\|_{L^{p}\left(\mathbb{F}^{* n}\right)}
$$

if and only if $r \leq k$ and $p \geq \frac{r+k}{k}$. Furthermore, if $r \leq k$ and $p=\frac{r+k}{k}$, we may take the constant $C$ to depend only on $n$ and $k$.

An example for the reader to bear in mind is $\mathfrak{p}_{1, a}(t)=a\left(t, t^{2}, \ldots, t^{n}\right)$ as $a$ varies over $\mathbb{F}$. The images are disjoint (except at 0 ) and the conclusion is $L^{p}$ boundedness for $p \geq 2$.

We shall derive these theorems from more general results; see Sections 2 and 3 below for necessary and sufficient conditions respectively. Some remarks on surfaces containing affine subspaces of high dimension are made in Section 4.

The main tools we shall use in proving these results are the standard tools of harmonic analysis (the Fourier transform, Littlewood-Paley theory - in a particularly primitive form, square function estimates) together with nontrivial estimates of A. Weil [11] for exponential sums over finite fields. These estimates are a consequence of Weil's resolution of the Riemann Hypothesis for curves in finite fields. In the present context they play the role of decay estimates for Fourier transforms of surface-carried measures that are so crucial in the euclidean case when the Fourier transform approach is used.

We also examine, in Section 5, a case of an averaging operator over $k$-dimensional surfaces in a non-convolution setting. Here the aproach is combinatorial.

We should emphasise that the novelty of our paper is not in the techniques involved, which are standard; rather in their extreme simplicity in this setting, leading to what seem to be very sharp results. Of course one must remember that this is only possible due to the results of Weil [11] which are fundamental in our approach. It would be of interest to find another approach to Theorems 1 and 2 which does not rely upon the results of [11].

1.1. Fourier transform and other notation. Let $\mathbb{F}$ be a finite field of characteristic $q \neq 2$. Let $e: \mathbb{F} \rightarrow S^{1}=\{z \in \mathbb{C}|| z \mid=1\}$ be a nonprincipal additive character of $\mathbb{F}$. (Thus, for example, if $\mathbb{F}=\mathbb{Z}_{q}, e(x)=\exp (2 \pi i x / q)$ defines such a character.) The vector space $\mathbb{F}^{n}$ is a locally compact abelian group with characters $e_{\xi}$ indexed by $\xi \in \mathbb{F}^{n *}$ (the dual group) and given by

$$
\begin{aligned}
e_{\xi}(x) & =e(x \cdot \xi)=e\left(x_{1} \xi_{1}+\ldots .+x_{n} \xi_{n}\right) \\
& =e_{\xi_{1}}\left(x_{1}\right) \ldots . . e_{\xi_{n}}\left(x_{n}\right)
\end{aligned}
$$

(Note that although we employ the "dot product" notation $x \cdot \xi$, there is no inner product structure here.) 
For $f: \mathbb{F}^{n} \rightarrow \mathbb{C}$ its Fourier transform $\hat{f}: \mathbb{F}^{n *} \rightarrow \mathbb{C}$ is defined by

$$
\begin{aligned}
\hat{f}(\xi) & =\sum_{x \in \mathbb{F}^{n}} f(x) e(-x \cdot \xi) \\
& :=\int_{\mathbb{F}^{n}} f(x) e(-x \cdot \xi) d x .
\end{aligned}
$$

Thus integration on $\mathbb{F}^{n}$ is with respect to un-normalised counting measure.

The Fourier inversion formula is

$$
\begin{aligned}
f(x) & =\frac{1}{|\mathbb{F}|^{n}} \sum_{\xi \in \mathbb{F}^{n *}} \hat{f}(\xi) e(x \cdot \xi) \\
& =\int_{\mathbb{F}^{n *}} \hat{f}(\xi) e(x \cdot \xi) d \xi
\end{aligned}
$$

We recall that integration on $\mathbb{F}^{n *}$ is with respect to normalised counting measure; the total mass of $\mathbb{F}^{n *}$ is 1 .

For a measure $\sigma_{\mathfrak{p}}$ associated to a polynomial $\mathfrak{p}$ as in the Introduction, its inverse Fourier transform is given by

$$
\sigma_{\mathfrak{p}}^{\vee}(x)=|\mathbb{F}|^{-k} \sum_{s \in \mathbb{F}^{k}} e(x \cdot \mathfrak{p}(s)) .
$$

We have the following standard results for $f$ and $g$ defined on $\mathbb{F}^{n *}$ :

- $\int_{\mathbb{F}^{n *}} \hat{f} \overline{\hat{g}}=\int_{\mathbb{F}^{n}} f \bar{g}$

- $\|\hat{f}\|_{L^{\infty}\left(\mathbb{F}^{n *}\right)} \leq\|f\|_{L^{1}\left(\mathbb{F}^{n}\right)}$

- $(f * g)^{\wedge}=\hat{f} \hat{g}$

- $(f g)^{\wedge}=\hat{f} * \hat{g}$

We shall use these results without further comment. We also note that

$$
\sum_{x \in \mathbb{F}} e(x)=0 .
$$

Finally, we caution that ' $\mathfrak{p}$ ' denotes a polynomial and ' $p$ ' an $L^{p}$-index. Occasionally ' $q$ ' will denote the characteristic of a field $\mathbb{F}$, but more often an $L^{q}$-index. The context will make clear which meaning is implied at each occurence. We use both notations $|\cdot|$ and \# to denote the cardinality of a set.

\section{NECESSARY CONDITIONS}


2.1. The averaging problem. Let $\mathfrak{p}: \mathbb{F}^{k} \rightarrow \mathbb{F}^{n *}$ be a polynomial of degree $d$. Let $\sigma=\sigma_{\mathfrak{p}}$ be the measure associated to $\mathfrak{p}$ as in the Introduction. We wish to determine for which $1 \leq p, q \leq \infty$ we have

$$
\|f * \sigma\|_{L^{q}\left(\mathbb{F}^{n *}\right)} \leq C\|f\|_{L^{p}\left(\mathbb{F}^{n *}\right)}
$$

with the constant $C$ depending possibly on $k, n, d$ and $\max _{\xi \in \mathbb{F}^{n *}} \# \mathfrak{p}^{-1}(\xi)$, but not upon $|\mathbb{F}|$ in any explicit way.

Since $\sigma$ has total mass 1, (4) always holds if $p=q$ by Young's inequality, with $C=1$. Since $\mathbb{F}^{n *}$ has total mass 1 it continues to hold with $C=1$ when $1 \leq q \leq$ $p \leq \infty$. So the main interest is what happens when $1 \leq p<q \leq \infty$. Let

$$
\begin{aligned}
f(\xi) & = \begin{cases}1 & \xi=0 \\
0 & \xi \neq 0\end{cases} \\
& =|\mathbb{F}|^{-n} \delta_{0}(\xi)
\end{aligned}
$$

(where $\delta_{0}$ is understood to have mass 1 ). Then

$$
\|f\|_{L^{p}\left(\mathbb{F}^{n *}\right)}=|\mathbb{F}|^{-n / p} .
$$

On the other hand,

$$
f * \sigma(\xi)=|\mathbb{F}|^{-n} \sigma(\xi)=|\mathbb{F}|^{-k} \# \mathfrak{p}^{-1}(\xi)
$$

so that

$$
\begin{aligned}
\|f * \sigma\|_{L^{q}\left(\mathbb{F}^{n *}\right)} & =|\mathbb{F}|^{-k}\left(\frac{1}{|\mathbb{F}|^{n}} \sum_{\xi} \# p^{-1}(\xi)^{q}\right)^{\frac{1}{q}} \\
& \geq|\mathbb{F}|^{-k}|\mathbb{F}|^{\frac{k-n}{q}}(\text { as } q \geq 1) .
\end{aligned}
$$

So in order for (4) to hold we must have

$$
|\mathbb{F}|^{-k+\frac{k-n}{q}} \leq C|\mathbb{F}|^{-\frac{n}{p}}
$$

Thus (4) can hold with $C$ independent of $|\mathbb{F}|$ only when

$$
\frac{n}{p} \leq k+\frac{n-k}{q} \text {. }
$$

By duality we obtain that if (4) holds with $C$ independent of $|\mathbb{F}|$, then $\left(\frac{1}{p}, \frac{1}{q}\right)$ must lie in the convex hull of the points $(0,1),(1,1),(0,0)$ and $\left(\frac{n}{2 n-k}, \frac{n-k}{2 n-k}\right)$. The last of these points is where the interest lies.

In the case that the image of $\mathfrak{p}$ contains an $s$-dimensional affine subspace of $\mathbb{F}^{n *}$, it makes sense to test (4) on the characteristic function of that $s$-plane, yielding the necessary condition $\frac{1}{q} \geq \frac{1}{p}-\frac{k-s}{n-s}$. (We leave the details of this calculation to the interested reader.) This provides a further necessary condition when $s>k / 2$. 
2.2. The maximal averaging problem. Let, for $\alpha \in \mathcal{A}, \mathfrak{p}_{\alpha}: \mathbb{F}^{k} \rightarrow \mathbb{F}^{n *}$ be a polynomial of degree at most $d$. Let $\sigma_{\alpha}$ be associated to $\mathfrak{p}_{\alpha}$ as in the Introduction. We wish to determine those exponents $p$ for which we have

$$
\left\|\sup _{\alpha \in \mathcal{A}}\left|f * \sigma_{\alpha}\right|\right\|_{p} \leq C\|f\|_{p}
$$

with $C$ depending on $k, n, d$ and $\max _{\alpha \in \mathcal{A}} \max _{\xi} \# \mathfrak{p}_{\alpha}^{-1}(\xi)$, as well as on the sizes of the indexing set $\mathcal{A}$ and of $\bigcup_{\alpha \in \mathcal{A}} \operatorname{im} \mathfrak{p}_{\alpha}$.

Define the number $r$ by $\#\left(\bigcup_{\alpha \in \mathcal{A}} \operatorname{im} \mathfrak{p}_{\alpha}\right)=|\mathbb{F}|^{k+r}$. Note that $|\mathbb{F}|^{r} \leq \# \mathcal{A}$, and that $r \leq n-k$. Take $f=|\mathbb{F}|^{-n} \delta_{0}$ as in the previous subsection. As before, $\|f\|_{p}=|\mathbb{F}|^{-n / p}$, while $f * \sigma_{\alpha}(\xi) \geq|\mathbb{F}|^{-k}$ on im $\mathfrak{p}_{\alpha}$, so that $\sup \left|f * \sigma_{\alpha}(\xi)\right| \geq|\mathbb{F}|^{-k}$ on $\bigcup_{\alpha \in \mathcal{A}} \operatorname{im} \mathfrak{p}_{\alpha}$. Thus

$$
\begin{aligned}
\left\|\sup _{\alpha}\left|f * \sigma_{\alpha}\right|\right\|_{p} & \geq|\mathbb{F}|^{-k}\left(\#\left(\bigcup_{\alpha \in \mathcal{A}} \operatorname{im} \mathfrak{p}_{\alpha}\right)|\mathbb{F}|^{-n}\right)^{\frac{1}{p}} \\
& =|\mathbb{F}|^{-n / p}|\mathbb{F}|^{-k+\frac{k+r}{p}} .
\end{aligned}
$$

Consequently if (6) is to hold with $C$ independent of $|\mathbb{F}|$ we must have $p \geq$ $\frac{k+r}{k}$. Obviously, when $p=\infty,(6)$ holds, so the main interest is what happens at $p=\frac{k+r}{k}$.

One may think of the index ' $r$ ' as measuring the "number of parameters" in the family of maximal averages: if $\# \operatorname{im} \mathfrak{p}_{\alpha}=|\mathbb{F}|^{k}$ for each $\alpha$ and the distinct im $\mathfrak{p}_{\alpha}$ are disjoint, then $\# \bigcup_{\alpha \in \mathcal{A}} \operatorname{im} \mathfrak{p}_{\alpha}=|\mathbb{F}|^{k} \# \mathcal{A}$. Our assumption then corresponds to $\# \mathcal{A}=$ $|\mathbb{F}|^{r}$. So $r=0$ corresponds to a simple convolution operator while $r=1$ corresponds to the one-parameter averages as employed for example in the Hardy-Littlewood and spherical maximal functions. Higher values of $r$ correspond to multiparameter averages. (In our later discussion on sufficient conditions, we shall always assume that each $\mathfrak{p}_{\alpha}$ is injective, so that $r$ will always be nonnegative.)

Now we discuss the other necessary condition $r \leq k$ from Theorem 2 .

Let $\mathfrak{p}: \mathbb{F}^{k} \rightarrow \mathbb{F}^{n *}$ be an arbitrary polynomial of degree $d$. We will let $\mathfrak{p}_{\alpha}$ be suitable translates of $\mathfrak{p}$ (i.e. we take $A_{\alpha}=0$ for all $\alpha$ ). Indeed, for $s \in \mathbb{F}^{m *}$ we define $\mathfrak{p}_{s}=\mathfrak{p}+(s, 0)$ and $E=\bigcup_{s^{\prime} \in \mathbb{F}^{(n-m) *}}\left\{\left(0, s^{\prime}\right)-\operatorname{im} \mathfrak{p}\right\}$. Then $|E| \leq|\mathbb{F}|^{n-m+k}$, and so $\left\|\chi_{E}\right\|_{p} \leq|\mathbb{F}|^{\frac{k-m}{p}}$. On the other hand, if for $y \in \mathbb{F}^{n *}$ we set $y=\left(s, s^{\prime}\right) \in$ $\mathbb{F}^{m *} \times \mathbb{F}^{(n-m) *}$, then $\sup _{s} \chi_{E} * \sigma_{s}(y)=1$. (We have $\chi_{E} * \sigma_{s}(y) \leq 1$ always, and with equality iff $\chi_{E}=1$ on $y-\operatorname{im} \mathfrak{p}_{s}=\left(s, s^{\prime}\right)-(\operatorname{im} \mathfrak{p}+(s, 0))=\left(0, s^{\prime}\right)-\operatorname{im} \mathfrak{p}$. But $E$ is the union of these, so that $\sup _{s} \chi_{E} * \sigma_{s}(y)=1$ for all $y \in \mathbb{F}^{n *}$.) Therefore $\left\|\sup _{s} \chi_{E} * \sigma_{s}\right\|_{p}=1$. Hence if (6) is to hold for this particular family of affine images of $\mathfrak{p}$ with $C$ independent of $|\mathbb{F}|$ we must have $m \leq k$.

In the situation of Theorem 2, we are assuming that the images of the $\mathfrak{p}_{\alpha}$ are disjoint, and so the role of $m$ in the previous paragraph is taken by $r$. 
It is to be noted that a similar phenomenon occurs in the euclidean case when we use translations. We do not know whether it is necessary that $r \leq k$ when we use only linear rather than affine images of a given fixed $\mathfrak{p}$.

\section{SufFiciEnt CONDitions}

We first discuss the main analytical arguments for the averaging and maximal averaging problems respectively, and then discuss the contribution made by Weil's estimates [11].

3.1. Averages. We begin with the main argument for Theorem 1.

Theorem 3. Let $1 \leq k<n$ and let $\mathfrak{p}: \mathbb{F}^{k} \rightarrow \mathbb{F}^{n *}$ be a polynomial (of degree $d$ ) such that for $x \neq 0$,

$$
\left|\sigma_{\mathfrak{p}}^{\vee}(x)\right| \leq(d-1)^{k}|\mathbb{F}|^{-k / 2}
$$

Then

$$
\left\|f * \sigma_{\mathfrak{p}}\right\|_{L^{\frac{2 n-k}{n-k}}\left(\mathbb{F}^{n *}\right)} \leq A\|f\|_{L^{\frac{2 n-k}{n}}\left(\mathbb{F}^{n *}\right)}
$$

where $A=1+(d-1)^{k \frac{2 n-2 k}{2 n-k}}\left[\max _{\xi} \# \mathfrak{p}^{-1}(\xi)\right]^{\frac{k}{2 n-k}}$.

\section{Remarks}

1. The key issue in applying this theorem is the verification of (7), in particular in the setting of Theorem 1. It is here that the analysis of Weil [11] enters and plays a decisive role. It is for this reason that the constant in (7) is written in the way it is. We discuss this in detail below.

2. Interpolation with trivial results gives the full range of exponents for which convolution with $\sigma_{\mathfrak{p}}$ is bounded with a constant independent of $|\mathbb{F}|$.

3. The second term appearing in $A$ is merely a convex combination of $(d-1)^{k}$ and $\max _{\xi} \# \mathfrak{p}^{-1}(\xi)$.

Proof. We write $\sigma_{\mathfrak{p}}$ as $\sigma$ for simplicity. We decompose $\sigma^{\vee}$ as

$$
\sigma^{\vee}=\sigma^{\vee} \chi_{x \neq 0}+\delta_{0}
$$

(recalling that $\sigma^{\vee}(0)=$ mass of $\sigma=1$ ). This is the Littlewood-Paley decomposition in the setting of vector spaces over finite fields.

Correspondingly we have

$$
\sigma=\hat{K}+1
$$

where $K(x)=\sigma^{\vee}(x) \chi_{x \neq 0}$ satisfies $\|K\|_{\infty} \leq(d-1)^{k}|\mathbb{F}|^{-k / 2}$ by assumption (7). Now

$$
\|f * 1\|_{q} \leq\|f\|_{p} \quad(1 \leq p, q \leq \infty),
$$

so it suffices to consider the contribution of convolution with $\hat{K}$. 
For this we do an $L^{1}-L^{\infty}$ and an $L^{2}-L^{2}$ estimate. For the former we have

$$
\|f * \hat{K}\|_{\infty} \leq\|\hat{K}\|_{\infty}\|f\|_{1}=\|\sigma-1\|_{\infty}\|f\|_{1} \leq|\mathbb{F}|^{n-k} \max _{\xi} \# \mathfrak{p}^{-1}(\xi)\|f\|_{1} ;
$$

while for the latter we have, by virtue of (7),

$$
\|f * \hat{K}\|_{2}=\left\|f^{\vee} K\right\|_{2} \leq\|K\|_{\infty}\left\|f^{\vee}\right\|_{2} \leq(d-1)^{k}|\mathbb{F}|^{-k / 2}\|f\|_{2} .
$$

Interpolation between these two estimates finishes the proof.

Further results on convolution averages of the type discussed here are to be found in $[6]$.

3.2. Maximal averages. We now give the main argument for Theorem 2. Once again the main issue will be verification of (7) in that setting.

Theorem 4. Let $1 \leq k<n$. Let $\mathcal{A}$ be an indexing set satisfying $\# \mathcal{A} \leq D|\mathbb{F}|^{r}$. For $\alpha \in \mathcal{A}$ suppose $\mathfrak{p}_{\alpha}: \mathbb{F}^{k} \rightarrow \mathbb{F}^{n *}$ is a polynomial such that (7) holds when $x \neq 0$ for all $\alpha$, and such that $\# \bigcup_{\alpha \in \mathcal{A}}$ im $\mathfrak{p}_{\alpha} \leq D|\mathbb{F}|^{k+\tilde{r}}$ for some $\tilde{r} \leq r$. If $r \leq k$, then

$$
\left\|\sup _{\alpha \in \mathcal{A}}\left|f * \sigma_{\alpha}\right|\right\|_{L^{\frac{2 \tilde{r}-r+k}{\tilde{r}-r+k}\left(\mathbb{F}^{n *}\right)}} \leq B\|f\|_{L^{\frac{2 \tilde{r}-r+k}{\tilde{r}-r+k}\left(\mathbb{F}^{n *}\right)}}
$$

where $B$ depends only upon $d, k, D$ and $\max \max _{\xi} \# \mathfrak{p}_{\alpha}^{-1}(\xi)$.

\section{Remarks}

1. This is the sharp estimate when $\tilde{r}=r$ as the remarks of Section 2 indicate.

2. Once again, the constant $B$ depends neither on $|\mathbb{F}|$ nor the dimension $n$, and in this case the $L^{p}$ exponent is also independent of $n$.

3. Note that (7) is invariant under affine transformations, so that any family of affine images of a single $\mathfrak{p}$ satisfying (7) will also satisfy it.

4. The theorem does not cover the maximal operator corresponding to $\mathfrak{p}_{a, b}(t)=$ $\left(a t, b t^{2}\right)$ as $a, b$ vary over $\mathbb{F}$.

Proof. As in Theorem 3 we write $\sigma_{\alpha}^{\vee}=\sigma_{\alpha}^{\vee} \chi_{x \neq 0}+\delta_{0}$ and $\sigma_{\alpha}=\hat{K}_{\alpha}+1$. Once again we have $\|f * 1\|_{p} \leq\|f\|_{p}$ for all $p$, so it is enough to show

$$
\left\|\sup _{\alpha \in \mathcal{A}}\left|f * \hat{K}_{\alpha}\right|\right\|_{\frac{2 \tilde{r}-r+k}{\tilde{r}-r+k}} \leq B\|f\|_{\frac{2 \tilde{r}-r+k}{\tilde{r}-r+k}} .
$$

When $r \leq k, 1 \leq \frac{2 \tilde{r}-r+k}{\tilde{r}-r+k} \leq 2$, and we obtain the desired estimate by interpolation between $p=1$ and $p=2$. 
For the $p=1$ estimate, we have for each $\alpha$ and $\xi$,

$$
\begin{aligned}
\left|\hat{K}_{\alpha}(\xi)\right| & =\left|\sigma_{\alpha}(\xi)-1\right| \\
& \leq|\mathbb{F}|^{n-k} \# \mathfrak{p}_{\alpha}^{-1}(\xi)+1 \\
& \leq M|\mathbb{F}|^{n-k} \chi_{\cup_{\alpha} \mathrm{im} \mathfrak{p}_{\alpha}}(\xi)+1
\end{aligned}
$$

where $M=\sup _{\alpha} \sup _{\xi} \# \mathfrak{p}_{\alpha}^{-1}(\xi)$, so that

$$
\int_{\mathbb{F}^{n *}} \sup _{\alpha}\left|\hat{K}_{\alpha}(\xi)\right| d \xi \leq 1+M|\mathbb{F}|^{-k} \# \bigcup_{\alpha} \operatorname{im} \mathfrak{p}_{\alpha}
$$

Thus,

$$
\begin{aligned}
\left\|\sup _{\alpha}\left|f * \hat{K}_{\alpha}\right|\right\|_{1} & \leq\left\||f| * \sup _{\alpha}\left|\hat{K}_{\alpha}\right|\right\|_{1} \\
& \leq\left[1+M|\mathbb{F}|^{-k} \#\left(\bigcup_{\alpha \in \mathcal{A}} \operatorname{im} \mathfrak{p}_{\alpha}\right)\right]\|f\|_{1} \\
& \leq\left[1+M D|\mathbb{F}|^{\tilde{r}}\right]\|f\|_{1} .
\end{aligned}
$$

Now we turn to the $p=2$ estimate. We have

$$
\begin{aligned}
\left\|\sup _{\alpha}\left|f * \hat{K}_{\alpha}\right|\right\|_{2} & \leq\left\|\left(\sum_{\alpha}\left|f * \hat{K}_{\alpha}\right|^{2}\right)^{\frac{1}{2}}\right\|_{2} \\
& =\left(\sum_{\alpha}\left\|f * \hat{K}_{\alpha}\right\|_{2}^{2}\right)^{\frac{1}{2}} \\
& \leq\left(\sum_{\alpha}\|\hat{f}\|_{2}^{2}\left\|K_{\alpha}\right\|_{\infty}^{2}\right)^{\frac{1}{2}} \\
& \leq(d-1)^{k}|\mathbb{F}|^{-k / 2}(\# \mathcal{A})^{\frac{1}{2}}\|f\|_{2} \\
& \leq(d-1)^{k} D^{\frac{1}{2}}|\mathbb{F}|^{\frac{r-k}{2}}\|f\|_{2} .
\end{aligned}
$$

Interpolation now shows that the bound on $L^{\frac{2 \tilde{r}-r+k}{\tilde{r}-r+k}}$ is essentially a convex combination of $D M$ and $(d-1)^{k} D^{\frac{1}{2}}$.

\section{Remark}

If, in the notation of Theorem $4, \# \mathcal{A}=|\mathbb{F}|^{r}$ and $\# \bigcup \operatorname{im} \mathfrak{p}_{\alpha}=|\mathbb{F}|^{k+\tilde{r}}$ with $\tilde{r}<r \leq$ $k$, the $L^{p}$ exponent $(2 \tilde{r}-r+k) /(\tilde{r}-r+k)$ is worse than $(\tilde{r}+k) / k$ which is what the analysis of the previous section suggests we should have. This is perhaps due to the inefficiency of estimating an $\ell^{\infty}$ norm by an $\ell^{2}$ one in the $p=2$ estimate.

Further results on maximal functions associated to quadric surfaces are to be found in $[6]$.

3.3. Exponential sums and decay estimates. We shall obtain the estmates (7) needed above by using Weil's remarkable estimates, see [11]. We use the notation $(\cdot, \cdot)$ to denote greatest common divisor. 
Theorem 5. Let $\mathbb{F}$ be a finite field and let $e: \mathbb{F} \rightarrow \mathbb{S}^{1}$ be a nonprincipal additive character. If $\mathfrak{p}: \mathbb{F} \rightarrow \mathbb{F}$ is a polynomial of degree $d$ with (char $\mathbb{F}, d)=1$, then

$$
\left|\sum_{s \in \mathbb{F}} e(\mathfrak{p}(s))\right| \leq(d-1)|\mathbb{F}|^{\frac{1}{2}} .
$$

Note that when $\mathfrak{p}$ is quadratic this is simply a gauss sum estimate. Also when $\mathbb{F}$ is the field of integers modulo a prime, the estimate holds for any nonconstant polynomial $\mathfrak{p}$.

Corollary 6. Let $\mathfrak{p}: \mathbb{F} \rightarrow \mathbb{F}^{n *}$ be a polynomial curve of degree $d \geq 2$ such that im $\mathfrak{p}$ lies in no proper affine subspace of $\mathbb{F}^{n *}$. If char $\mathbb{F}>d$, and $x \neq 0$, then

$$
\left|\sigma_{\mathfrak{p}}^{\vee}(x)\right| \leq(d-1)|\mathbb{F}|^{-\frac{1}{2}}
$$

We can combine this corollary with Theorem 3 to obtain:

Corollary 7. Let $\mathfrak{p}: \mathbb{F} \rightarrow \mathbb{F}^{n *}$ be an injective polynomial curve of degree $d \geq 2$ such that im $\mathfrak{p}$ lies in no proper affine subspace of $\mathbb{F}^{n *}$. If char $\mathbb{F}>d$, then

$$
\left\|f * \sigma_{\mathfrak{p}}\right\|_{L^{\frac{2 n-1}{n-1}\left(\mathbb{F}^{n *}\right)}} \leq A\|f\|_{L^{\frac{2 n-1}{n}}\left(\mathbb{F}^{n *}\right)}
$$

where $A$ depends only on $n$ and $d$.

A similar remark applies in the context of maximal functions.

When $k \geq 2$, for reasons allied to Remark 3 below, we are not in a position to make such a general statement, and instead work with a more restricted class of polynomial surfaces.

We define $\mathfrak{p}: \mathbb{F}^{k} \rightarrow \mathbb{F}^{n *}$ as follows. Let $\mathfrak{q}_{j}^{i}: \mathbb{F} \rightarrow \mathbb{F}$ be a polynomial, where $1 \leq i \leq$ $n-k+1$ and $1 \leq j \leq k$, and where $\operatorname{deg} \mathfrak{q}_{j}^{i}>\operatorname{deg} \mathfrak{q}_{j}^{i-1} \geq 2$. For $t=\left(t_{1}, t_{2}, \ldots ., t_{k}\right) \in$ $\mathbb{F}^{k}$ let

$$
\mathfrak{p}(t)=\left(t_{1}, \ldots, t_{k}, \mathfrak{q}_{1}^{1}\left(t_{1}\right)+\ldots .+\mathfrak{q}_{k}^{1}\left(t_{k}\right), \ldots, \mathfrak{q}_{1}^{n-k+1}\left(t_{1}\right)+\ldots .+\mathfrak{q}_{k}^{n-k+1}\left(t_{n}\right)\right) .
$$

In Theorems 1 and 2 we consider the 'typical' case

$$
\mathfrak{p}_{k}(t)=\left(t_{1}, \ldots, t_{k}, t_{1}^{2}+\ldots+t_{k}^{2}, \ldots, t_{1}^{n-k+1}+\ldots+t_{k}^{n-k+1}\right) .
$$

Note that by construction, im $\mathfrak{p}$ lies in no proper affine subspace of $\mathbb{F}^{n *}$.

Proposition 8. Let the polynomial $\mathfrak{p}$ be as above. Suppose $\operatorname{char}(\mathbb{F})>\operatorname{deg} \mathfrak{p}=$ d. Then, for $x \neq 0$,

$$
\left|\sigma_{\mathfrak{p}}^{\vee}(x)\right| \leq(d-1)^{k}|\mathbb{F}|^{-k / 2} .
$$

Remark In order for (7) to hold it is necessary that im $\mathfrak{p}$ lie in no proper affine subspace of $\mathbb{F}^{n *}$, since if $x$ is such that $x \cdot \mathfrak{p}(t)=\beta$ for all $t$ we have $\sigma_{\mathfrak{p}}^{\vee}(x)=e(\beta)$.

Proof. We have

$$
\begin{aligned}
x \cdot \mathfrak{p}(t) & =x_{1} t_{1}+x_{k+1} \mathfrak{q}_{1}^{1}\left(t_{1}\right)+\ldots+x_{n} \mathfrak{q}_{1}^{n-k+1}\left(t_{1}\right) \\
& +x_{2} t_{2}+x_{k+1} \mathfrak{q}_{2}^{1}\left(t_{2}\right)+\ldots+x_{n} \mathfrak{q}_{2}^{n-k+1}\left(t_{2}\right) \\
& \vdots \\
& +x_{k} t_{k}+x_{k+1} \mathfrak{q}_{k}^{1}\left(t_{k}\right)+\ldots+x_{n} \mathfrak{q}_{k}^{n-k+1}\left(t_{k}\right)
\end{aligned}
$$


so that $\sum_{t \in \mathbb{F}^{k}} e(x \cdot \mathfrak{p}(t))$ factorises as the product

$$
\prod_{j=1}^{k} \sum_{s \in \mathbb{F}} e\left(x_{j} s+x_{k+1} \mathfrak{q}_{j}^{1}(s)+\ldots+x_{n} \mathfrak{q}_{j}^{n-k+1}(s)\right) .
$$

If now $x \neq 0$ but $x_{k+1}, x_{k+2}, \ldots, x_{n}$ are all zero, at least one of the factors is a nonprincipal character sum and hence is zero by (3). If some $x_{\ell} \neq 0, k+1 \leq \ell \leq n$ we can apply Weil's Theorem 5 to each factor to conclude that its absolute value is less than or equal to $\left(\operatorname{deg} \mathfrak{q}_{j}^{n-k+1}-1\right)|\mathbb{F}|^{\frac{1}{2}} \leq(d-1)|\mathbb{F}|^{\frac{1}{2}}$. Hence for $x \neq 0$,

$$
\left|\sum_{t \in \mathbb{F}^{k}} e(x \cdot \mathfrak{p}(t))\right| \leq(d-1)^{k}|\mathbb{F}|^{k / 2},
$$

from which (3) follows upon dividing by $|\mathbb{F}|^{k}$.

\section{Remarks}

1. Combining Theorems 3 and 4 with Proposition 8 we conclude the proofs of Theorems 1 and 2 respectively.

2. That the power of $|\mathbb{F}|$ in Proposition 8 is sharp can be seen (in special cases) by applying the necessary conditions of Section 2. More generally, a direct $L^{2} \operatorname{argument}$ is available. Indeed, assume that $\left|\sigma^{\vee}(x)\right| \leq A|\mathbb{F}|^{-\alpha / 2}$ when $x \neq 0$. Then $\left\|\sigma^{\vee}\right\|_{2}^{2} \leq$ $A^{2}|\mathbb{F}|^{-\alpha}\left(|\mathbb{F}|^{n}-1\right)+1 \approx|\mathbb{F}|^{n-\alpha}$, while $\|\sigma\|_{2}^{2}=\frac{1}{|\mathbb{F}|^{n}} \sum_{\xi \in \mathbb{F}^{n *}}|\mathbb{F}|^{2(n-k)} \# \mathfrak{p}^{-1}(\xi)^{2} \geq$ $|\mathbb{F}|^{n}(\# \text { im } \mathfrak{p})^{-1}$ (by Cauchy-Schwarz). So $|\mathbb{F}|^{n-\alpha} \geq C|\mathbb{F}|^{n}(\# \text { im } \mathfrak{p})^{-1}$. Hence if $\mathfrak{p}: \mathbb{F}^{k} \rightarrow \mathbb{F}^{n *}$, we have $|\mathbb{F}|^{\alpha} \leq C \# \operatorname{im} \mathfrak{p} \leq C|\mathbb{F}|^{k}$. So the best $\alpha$ in this case is $k$.

3. Deligne [4] has proved a far reaching $k$-dimensional generalisation of Weil's theorem, but it seems not so straightforward in practice to work directly with it in our context. However when $k=n-1$ and $\mathfrak{p}$ is of the form $\left(t_{1}, \ldots, t_{n-1}, \mathfrak{q}\left(t_{1}, \ldots, t_{n-1}\right)\right)$, with $\mathfrak{q}$ a quadratic form of full rank, direct calculation shows that (7) holds provided that char $\mathbb{F}>2$. (Just complete the square and find a product of gauss sums.)

\section{Averages over SURfaCes With AFFine subspaces of LARGE Dimension}

Let $\mathfrak{p}: \mathbb{F}^{k} \rightarrow \mathbb{F}^{n *}$ be a polynomial surface. Suppose im $\mathfrak{p}$ contains an affine subspace of dimension $s$. As we have seen in Section 2 above, when $s>k / 2$ this introduces the new necessary condition $\frac{1}{q} \geq \frac{1}{p}-\frac{k-s}{n-s}$ for the $L^{p}-L^{q}$ mapping problem. (This is in addition to the standard necessary conditions $\frac{1}{q} \geq \frac{1}{p}-\frac{n-k}{n}$ and $\frac{1}{q} \geq \frac{1}{n-k}\left(\frac{n}{p}-k\right)$.) One will therefore not have the optimal decay rate $\left|\sigma_{\mathfrak{p}}^{\vee}(x)\right| \leq$ $C|\mathbb{F}|^{-k / 2} \quad(x \neq 0)$ in such cases. Indeed, the proof of Theorem 3 together with the necessary condition $\frac{1}{q} \geq \frac{1}{p}-\frac{k-s}{r-s}$ yields:

Proposition 9. If $\mathfrak{p}: \mathbb{F}^{k} \rightarrow \mathbb{F}^{n *}$ is a polynomial surface such that $\left|\sigma_{\mathfrak{p}}^{\vee}(x)\right| \leq$ $C|\mathbb{F}|^{-r}$ for $x \neq 0$ and if the image of $\mathfrak{p}$ contains an affine $s$-dimensional subspace, then $r \leq k-s$. 
One might therefore ask whether, for each $n, k$ and $s$ with $0 \leq s \leq k<n$, there exist polynomial surfaces $\mathfrak{p}: \mathbb{F}^{k} \rightarrow \mathbb{F}^{n *}$ which contain an affine subspace of dimension $s$ and which satisfy

$$
\left|\sigma_{\mathfrak{p}}^{\vee}(x)\right| \leq C\left\{\begin{array}{cc}
|\mathbb{F}|^{-k / 2} & s \leq k / 2 \\
|\mathbb{F}|^{-(k-s)} & s \geq k / 2
\end{array}\right.
$$

for $x \neq 0$. If so, one may further ask what are the $L^{p}-L^{q}$ mapping properties of convolution with $\sigma_{\mathfrak{p}}$ when $s>k / 2$.

To partially answer the first question when $s \geq k / 2$, take $\ell$ even, $\ell \leq k$ and consider the polynomial

$\left(t_{1}, \ldots, t_{k}, t_{1}^{2}-t_{2}^{2}+t_{3}^{2}-t_{4}^{2}+\ldots+t_{\ell-1}^{2}-t_{\ell}^{2}, \ldots \ldots ., t_{1}^{n-k+1}-t_{2}^{n-k+1}+\ldots .+t_{\ell-1}^{n-k+1}-t_{\ell}^{n-k+1}\right)$.

The image of this map contains the subspace

$$
\left(\alpha_{1},-\alpha_{1}, \alpha_{2},-\alpha_{2}, \ldots ., \alpha_{\ell / 2},-\alpha_{\ell / 2}, \alpha_{\ell+1}, \ldots \alpha_{k}, 0, \ldots 0\right)
$$

of dimension $\ell / 2+(k-\ell)=k-\ell / 2$, while by the results of Section 3 , the corresponding $\sigma_{\mathfrak{p}}$ satisfies $\left|\sigma_{\mathfrak{p}}^{\vee}(x)\right| \leq(n-k)^{k}|\mathbb{F}|^{-\ell / 2}$ when $x \neq 0$. Setting $s=k-\ell / 2$ gives the desired estimate.

To partially answer the second question, once again tracing the proof of Theorem 3 we obtain that

$$
\left\|f * \sigma_{\mathfrak{p}}\right\|_{q} \leq C\|f\|_{p}
$$

for $\left(\frac{1}{p}, \frac{1}{q}\right)=\left(\frac{n-k}{2(n-s)}, \frac{n-2 s+k}{2(n-s)}\right.$ ) (which is the intersection of $\frac{1}{q}=\frac{1}{p}-\frac{k-s}{n-s}$ with $\frac{1}{p}+\frac{1}{q}=1$.) To obtain a further partial result, we introduce the concept of Sobolev spaces over vector spaces over finite fields. For $1 \leq p \leq \infty$ and $\alpha \geq 0$ we define the $L_{\alpha}^{p}$ norm of $f$ on $\mathbb{F}^{n *}$ by

$$
\|f\|_{L_{\alpha}^{p}}=\|f\|_{p}+|\mathbb{F}|^{\alpha}\left\|f-\int_{\mathbb{F}^{n *}} f\right\|_{p} .
$$

Analogues of the usual Sobolev embedding and interpolation theorems hold, and are left as an exercise for the interested reader. The size estimate for $\sigma_{\mathfrak{p}}^{\vee}$ gives

$$
\left\|f * \sigma_{\mathfrak{p}}\right\|_{L_{k-s}^{2}} \leq C\|f\|_{2}
$$

and we trivially have

$$
\left\|f * \sigma_{\mathfrak{p}}\right\|_{\infty} \leq\|f\|_{\infty}
$$

so that for $0 \leq \frac{1}{p} \leq \frac{1}{2}$

$$
\left\|f * \sigma_{\mathfrak{p}}\right\|_{\frac{L^{p(k-s)}}{p}} \leq C\|f\|_{p} .
$$

By Sobolev embedding,

$$
\left\|f * \sigma_{\mathfrak{p}}\right\|_{q} \leq C\left\|f * \sigma_{\mathfrak{p}}\right\|_{\frac{L^{p} p(k-s)}{p}} \leq C\|f\|_{p}
$$

provided

$$
\frac{1}{q}=\frac{1}{p}-\frac{2(k-s)}{p n}=\frac{n-2 k+2 s}{p n}
$$


and $0 \leq \frac{1}{p} \leq \frac{1}{2}$. This represents an improvement over what we obtain by trivially interpolating the points $(0,0),\left(\frac{n-k}{2(n-s)}, \frac{n-2 s+k}{2(n-s)}\right)$, but does not give any information on the line $\frac{1}{q}=\frac{1}{p} \frac{n-k}{n}$, (where the examples lead us to believe that we should have estimates for sufficiently small $1 / p$.)

\section{RADON TRANSFORMS}

For $1 \leq k \leq n-1$, let $G_{n, k}$ be the class of all $k$-planes in $\mathbb{F}^{n *}$. For $f$ defined on $\mathbb{F}^{n *}$ and $\omega \in G_{n, k}$ let

$$
R f(\omega)=\frac{1}{|\mathbb{F}|^{k}} \sum_{\xi \in \omega} f(\xi)
$$

We consider this as a map from $L^{p}\left(\mathbb{F}^{n *}\right)$ to $L^{q}\left(G_{n, k}\right)$ endowed with normalised counting measure. The following result is a finite field analogue of one of Christ's result in $[2]$

Theorem 10. If

$$
\|R f\|_{L^{q}\left(G_{n, k}\right)} \leq C\|f\|_{L^{p}\left(\mathbb{F}^{n *}\right)}
$$

holds with $C$ independent of $|\mathbb{F}|$, then $\left(\frac{1}{p}, \frac{1}{q}\right)$ lies in the convex hull $\mathcal{H}$ of $\left(\frac{k+1}{n+1}, \frac{1}{n+1}\right)$, $(0,0),(1,1)$ and $(0,1)$. Conversely, if $\left(\frac{1}{p}, \frac{1}{q}\right)$ lies in $\mathcal{H} \backslash\left(\frac{k+1}{n+1}, \frac{1}{n+1}\right)$, then (8) holds with $C$ independent of $|\mathbb{F}|$. Finally, if $\left(\frac{1}{p}, \frac{1}{q}\right)=\left(\frac{k+1}{n+1}, \frac{1}{n+1}\right)$, then the restricted type inequality

$$
\|R f\|_{L^{n+1}\left(G_{n, k}\right)} \leq C\|f\|_{L^{\frac{n+1}{k+1}, 1}\left(\mathbb{F}^{n *}\right)}
$$

holds with $C$ independent of $|\mathbb{F}|$.

It will be useful to note that the dual operator $R^{*}$ to $R$ is the map taking functions $g$ defined on $G_{n, k}$ to functions defined on $\mathbb{F}^{n *}$ given by

$$
R^{*} g(\xi)=\frac{1}{P_{n, k}} \sum_{\{\omega: \xi \in \omega\}} g(\omega)
$$

where $P_{n, k}$ denotes the number of $k$-planes containing a given point, and that there is a corresponding dual inequality

$$
\left\|R^{*} g\right\|_{L^{p^{\prime}\left(\mathbb{F}^{n *}\right)}} \leq C\|g\|_{L^{q^{\prime}}\left(G_{n, k}\right)}
$$

to (8) and dual statement to Theorem 10. This remark suggests also the general problem of Radon transforms mapping $L^{p}\left(G_{n, \ell}\right)$ to $L^{q}\left(G_{n, k}\right)$ for various $0 \leq k, \ell \leq$ $n-1$; some results in this setting are to be found in [6].

Before we begin the proof we first note that $\left|G_{n, k}\right|$ is bounded above and below by an absolute constant times $|\mathbb{F}|^{(n-k)(k+1)}$, and that the number $P_{n, k}$ of $k$-planes containing any given point is bounded above and below by an absolute constant times $|\mathbb{F}|^{k(n-k)}$. Moreover the number of $k$-planes containing a given $s$-plane (with $s \leq k)$ is bounded above and below by $|\mathbb{F}|^{(n-k)(k-s)}$. 
Proof. We begin with the necessary conditions.

Taking $f$ to be the characteristic function of 0 , we have $\|f\|_{p}=|\mathbb{F}|^{-n / p}$. Now $R f(\omega)=|\mathbb{F}|^{-k}$ if $0 \in \omega$ and is zero otherwise. So

$$
\|R f\|_{q}=|\mathbb{F}|^{-k}\left(\#\{\mathrm{k} \text {-planes containing } 0\} /\left|G_{n, k}\right|\right)^{1 / q}
$$

which is bounded above and below by $|\mathbb{F}|^{-(k+(n-k) / q)}$. So a necessary condition is $k+(n-k) / q \geq n / p$.

For a second necessary condition we take $g$ to be the characteristic function of a single $k$-plane $V$ in the dual inequality (10). Now $\|g\|_{q^{\prime}}=\left|G_{n, k}\right|^{-1 / q^{\prime}}$, which is bounded above and below by $|\mathbb{F}|^{-(n-k)(k+1) / q^{\prime}}$. Now $R^{*} g(\xi)=P_{n, k}^{-1}$ if $\xi \in V$ and is zero otherwise. So

$$
\left\|R^{*} g\right\|_{p^{\prime}}=P_{n, k}^{-1}|\mathbb{F}|^{-(n-k) / p^{\prime}}
$$

which is bounded above and below by $|\mathbb{F}|^{-(n-k) k}|\mathbb{F}|^{-(n-k) / p^{\prime}}=|\mathbb{F}|^{-(n-k)\left(k+1 / p^{\prime}\right)}$. So a second necessary condition is $\left(k+1 / p^{\prime}\right) \geq(k+1) / q^{\prime}$, i.e. $1 / q \geq 1 /(k+1) p$.

Noting that the lines $1 / q=1 /(k+1) p$ and $k+(n-k) / q=n / p$ meet at $\left(\frac{1}{p}, \frac{1}{q}\right)=$ $\left(\frac{k+1}{n+1}, \frac{1}{n+1}\right)$ we see the necessity of $\left(\frac{1}{p}, \frac{1}{q}\right)$ lying in $\mathcal{H}$.

Now we turn to the sufficient conditions. Since inequality (8) is easily seen to hold for $\left(\frac{1}{p}, \frac{1}{q}\right)=(0,0),(1,1)$ and $(0,1)$, it suffices by interpolation to prove $(9)$, which is (8) applied to $f$ of the form $\chi_{E}$, and amounts to showing

$$
\sum_{\omega \in G_{n, k}} R \chi_{E}(\omega)^{n+1} \leq C\left(\frac{|E|}{|\mathbb{F}|^{n}}\right)^{k+1}\left|G_{n, k}\right| .
$$

Upon multiplying out the left hand side and using $\left|G_{n, k}\right| \sim|\mathbb{F}|^{(n-k)(k+1)}$ this becomes

$$
\sum_{\xi_{1}, \ldots, \xi_{n+1} \in E} \# k \text {-planes containing } \xi_{1}, \ldots, \xi_{n+1} \leq C|E|^{k+1}|\mathbb{F}|^{k(n-k)} .
$$

For $0 \leq s \leq k$, let $\Delta_{s}$ consist of the $(n+1)$-tuples of points $\left(\xi_{1}, \ldots, \xi_{n+1}\right) \in E^{n+1}$ such that their affine span $V\left(\xi_{1}, \ldots, \xi_{n+1}\right)$ is of dimension $s$. Then,

$$
\begin{aligned}
& \sum_{\xi_{1}, \ldots, \xi_{n+1} \in E} \# k \text {-planes containing } \xi_{1}, \ldots, \xi_{n+1} \\
= & \sum_{s=0}^{k} \sum_{\left(\xi_{1}, \ldots, \xi_{n+1}\right) \in \Delta_{s}} \# k \text {-planes containing } \xi_{1}, \ldots, \xi_{n+1} \\
= & \sum_{s=0}^{k} \sum_{V} \sum_{\left(\xi_{1}, \ldots, \xi_{n+1}\right) \in \Delta_{s}: V\left(\xi_{1}, \ldots, \xi_{n+1}\right)=V} \# k \text {-planes containing } V \\
\leq & C|\mathbb{F}|^{(n-k)(k-s)} \sum_{s=0}^{k} \sum_{V} \#\left\{\left(\xi_{1}, \ldots, \xi_{n+1}\right) \in \Delta_{s}: V\left(\xi_{1}, \ldots, \xi_{n+1}\right)=V\right\},
\end{aligned}
$$


so in order to show (11) it suffices to show, for each $0 \leq s \leq k$,

$$
\sum_{V} \#\left\{\left(\xi_{1}, \ldots, \xi_{n+1}\right) \in \Delta_{s}: V\left(\xi_{1}, \ldots, \xi_{n+1}\right)=V\right\} \leq C|E|^{k+1}|\mathbb{F}|^{(n-k) s},
$$

that is,

$$
\# \Delta_{s} \leq C|E|^{k+1}|\mathbb{F}|^{(n-k) s} .
$$

Now the left hand side of (12) does not see the parameter $k$, and the right hand side has lower bounds (when $k \in\{s, \ldots, n-1\}$ ) of $|E|^{s+1}|\mathbb{F}|^{s(n-s)}$ when $|E| \geq|F|^{s}$, and $|E|^{n}|\mathbb{F}|^{s} \geq|E|^{n+1}$ when $|E| \leq|F|^{s}$ respectively. In the latter case $|E| \leq|F|^{s}$ we are finished and so we are left with showing

$$
\# \Delta_{s} \leq C|E|^{s+1}|\mathbb{F}|^{s(n-s)}
$$

when $|E| \geq|F|^{s}$.

Now $\# \Delta_{s} \leq\left(\begin{array}{c}n+1 \\ s+1\end{array}\right) \# \tilde{\Delta}_{s}$, where $\tilde{\Delta}_{s}$ consists of those members $\left(\xi_{1}, \ldots, \xi_{n+1}\right)$ of $\Delta_{s}$ such that the affine span of $\xi_{1}, \xi_{2} \ldots, \xi_{s+1}$ is of dimension $s$. Thus for each of the first $s+1$ coordinates of a member of $\tilde{\Delta}_{s}$ we choose from amongst at most $|E|$ possibilities, leading to a factor of $|E|^{s+1}$, while for $s+2 \leq j \leq n+1$ we are constrained to choose $\xi_{j}$ from the $s$-dimensional affine plane already determined by the first $s+1$ choices. That is, for $s+2 \leq j \leq n+1$ there are at most $|\mathbb{F}|^{s}$ choices for $\xi_{j}$, leading to a factor of $|\mathbb{F}|^{s(n-s)}$. Altogether, $\# \tilde{\Delta}_{s} \leq|E|^{s+1}|\mathbb{F}|^{s(n-s)}$, so we arrive at (13) and this finishes the proof.

\section{FURTHER REMARKS}

Results such as Theorem 10 and Proposition 8 in the current paper have applications to the theory of restriction of the Fourier transform in the setting of vector spaces over finite fields, the main topic of [5]. For example, Proposition 8 together with the methods of [5] yields:

Proposition 11. With $\mathfrak{p}: \mathbb{F}^{k} \rightarrow \mathbb{F}^{n *}$ satisfying (7), and with

$$
(g d \sigma)^{\vee}(x)=\frac{1}{|\mathbb{F}|^{k}} \sum_{t \in \mathbb{F}^{k}} g(t) e(x \cdot \mathfrak{p}(t)),
$$

then

$$
\left\|(g d \sigma)^{\vee}\right\|_{L^{\frac{2(2 n-k)}{k}\left(\mathbb{F}^{n}\right)}} \leq C\|g\|_{L^{2}\left(\mathbb{F}^{k *}\right)}
$$

with $C$ independent of $|\mathbb{F}|$.

A Radon transform argument allows one to improve the restricted type $L^{8 / 5,1}$ extension theorem for paraboloids in $\mathbb{F}^{3 *}$ from [5] to the corresponding strong type result. Details will appear elsewhere.

Finally, two questions which we do not pursue here: which are the $L^{p}-L^{q}$ and Sobolev estimates for the maximal function of Theorem 2? And what is the effect of affine subspaces of large dimension on the maximal function of Theorem 2 ? 


\section{REFERENCES}

[1] J. Bourgain, N. Katz and T. Tao, A sum-product estimate in finite fields, and applications, Geom. Funct. Anal. 14 no. 1, (2004) 27-57.

[2] M. Christ, Estimates for the $k$-plane transform, Indiana U. Math. Jour. 33, no. 6, (1984) 891-910.

[3] M. Christ, Convolution, curvature, and combinatorics: a case study, Internat. Math. Res. Notices 19, (1998) 1033-1048.

[4] P. Deligne, La conjecture de Weil, I Inst. Hautes Etudes Sci. Publ. Math. 43, (1974) 273-307.

[5] G. Mockenhoupt and T. Tao, Restriction and Kakeya phenomena for finite fields, Duke Math. Jour. 121 2, (2004), 35-74.

[6] B. Stones, Three aspects of harmonic analysis over finite fields, Ph.D. thesis, University of Edinburgh, 2005.

[7] W. Schlag, On continuum incidence problems related to harmonic analysis, Jour. Funct. Anal. 201 2, (2003) 480-521.

[8] A. Seeger, Degenerate Fourier integral operators in the plane, Duke Math. Jour. 71, (1993) 685-745.

[9] E. M. Stein, Harmonic Analysis, Princeton University Press (1993).

[10] T. Tao and J. Wright, $L^{p}$-improving estimates for averages along curves, Jour. Amer. Math. Soc. 16, (2003) 605-638.

[11] A. Weil, On some exponential sums, Proc. Nat. Acad. Sci. U.S.A. 34, (1948) $204-207$.

[12] T. Wolff, Recent work connected with the Kakeya problem, Prospects in mathematics (Princeton, NJ, 1996), Amer. Math. Soc., Providence, RI, (1999) 129-162.

Anthony Carbery, School of Mathematics, University of Edinburgh, JCMB, King's Buildings, Mayfield Road, Edinburgh, EH9 3JZ, Scotland.

E-mail address: A.Carbery@ed.ac.uk

Brendan Stones, The John Henry Newman School, Hichin Road, Stevenage, HertfordSHIRE, SG1 4AE

E-mail address: brendan_maths@postmaster.co.uk

James Wright, School of Mathematics, University of Edinburgh, JCMB, King's Buildings, Mayfield Road, Edinburgh, EH9 3JZ, Scotland.

E-mail address: J.R.Wright@ed.ac.uk 